\title{
Double Crossed Syndrome in Cricketer's Shoulder: RCT
}

\author{
Sharma ${ }^{1}$, M., Jeba ${ }^{2}$, C. and Khatri ${ }^{3}$, S. \\ ${ }^{1}$ Post Graduate Student, ${ }^{2}$ Assistant Professor, ${ }^{3}$ Professor and Principal, \\ K.L.E.S. Institute of Physiotherapy, J. N. Medical College Campus, Belgaum 590 010, Karnataka INDIA
}

\section{Abstract}

The purpose of the study was to identify the effect of stretching and strengthening exercises on double crossed syndrome in cricket bowlers. This study was carried out at Belgaum Cricket Club and Union Gymkhana, Belgaum. The sample consisted of 30 male fast bowlers with double crossed syndrome. Their demographic data was collected and participants were randomly allocated into two groups (Study \& Control) of 15 each after obtaining their informed consent. Pre-interventional measurements were taken in terms of Forward Head Posture (FHP), Forward Shoulder Posture (FSP), Head to Shoulder Translation (HST), Head to Ankle Translation (HAT), Shoulder to Ankle Translation (SAT), New York Postural Rating Scale (NYPRS). Shoulder stretching and strengthening exercises were carried out using a theraband for a period of six weeks, following which post interventional measurements were taken. Interventional group showed statistically significant differences in terms of FSP, HST, SAT, NYPRS when compared with the control group. It is concluded that shoulder stretching and strengthening exercises are effective in reducing the muscular imbalance pattern in double crossed syndrome.

Key Words: Cricket bowlers, Forward shoulder posture, NYPRS.

\section{Introduction}

Cricket is one of the major international sports played in more than 60 countries. In India, cricket has always been much more than a sport. Although a non-contact sport, injuries in cricket are common and have been documented as far back as 1751 (Brasch, 1971). In 1970, cricket was regarded as a sport of 'Moderate Risk Injuries'. These days' cricketers are more susceptible to high risk injuries and cricket ranks $5^{\text {th }}$ among causes of non-fatal accidents, because today players are expected to train themselves longer, harder and earlier in life, to excel in sport (Weightman \& Browne, 1971). Cricket bowlers like other throwing sports involve repeated forceful ballistic arm actions which will put a great deal of eccentric load on the shoulder rotator cuff muscles predisposing them to injuries (Stretch, 2001). Bowling has been found to be the major cause of cricket injuries with $38 \%$ to $47.4 \%$ of schoolboy bowlers sustaining injuries in cricket (Stretch, 1995 \& Honcock \& Hawkins, 1996). Scapular instability is found in as many as $68 \%$ of rotator cuff problems and 100\% glenohumeral problems. The abnormal scapular biomechanics that occur as a result of this dysfunction create imbalance between agonist and antagonist muscles and predispose the shoulder to injuries (Voight \& Thomson, 2000).

To achieve peak performance during overhead activity, there must be optimal balance between mobility and stability. Most of the shoulder problems are due to improper technique and unbalanced upper body workouts. In highly trained athletes, an adaptation of increased activation of agonist over antagonist muscles has been reported in both upper and lower extremities. As a result of these neuromuscular and strength adaptations, the commonly observed forward head and rounded shoulder in bowlers develop overtime (Kluemper et al, 2006). Possibly this phenomena is 
caused by the stronger internal rotator and adductor muscles pulling the clavicle and scapula forward over the weaker external rotator and abductor muscles, leading to the forward shoulder posture (Kluemper et al, 2006).

Wide varieties of physiotherapy approaches have been suggested for strengthening and stretching of rotator cuff muscles for the correction of forward shoulder posture. Kendall et al (1993) and Suhrmann (2002) suggested that stretching of tight muscles and strengthening of the antagonist muscles is the appropriate intervention for correcting this positional or postural fault caused by muscle imbalance. If this forward shoulder posture can be addressed through a program of stretching and strengthening, perhaps the risk of shoulder injuries can be reduced.

The purpose of the study was to evaluate the effectiveness of six weeks stretching and strengthening program on forward shoulder posture in young competitive fast bowlers.

\section{Materials and Methods}

Thirty male high school and college age competitive cricket fast bowlers from Belgaum Cricket Club and Union Gymkhana, Belgaum served as subjects in the study. Subjects were included in this study if they fulfilled the following criteria:

- $\quad$ Cricket fast bowlers with forward shoulder posture, without shoulder pain, with no restriction in bowling and no restriction in training as a result of shoulder pain.

Subjects with recent humerus fractures, recurrent shoulder dislocation, recent shoulder surgeries, neurological pain like cervical radiculopathy and bowlers with any other associated problems other than that of forward shoulder posture (rotator cuff tendonitis, impingement syndrome etc) were not included.

\section{Testing procedure}

Measurements of both the right and left shoulder of each subject were taken before and after a 6 -week stretching and strengthening program. A device known as carpenter's trisquare and New York Postural Rating Scale (only shoulder component) was used for the measurements.

Participants were asked to stand the way they usually do (without footwear). A plumb line was hung vertically slightly anteriorly to the ankle in the saggital plane. The posture of the shoulder was then scored as per New York Postural Rating Scale (NYPRS) and the scoring was given as 5,3 or 1 as described by Arnold et al (2000). For the next measurement, participants were asked to stand with their back toward the wall without leaning on the wall. Various measurements were taken (from wall till tragus of ear, from wall till $2 \mathrm{cms}$ below the acromion process, from the wall till lateral most aspect of the lateral malleolus).

\section{Exercise Programme}

Six weeks of stretching and strengthening program along with normal training was administered to the interventional group after recording the initial measurements. At the first session, the participants were introduced to the different levels of theraband exercise bands. These bands are colour coded with each colour representing a different resistance. The participants were given 
the opportunity to do 5-repetitions of each exercise with several levels of theraband in order to estimate which level was appropriate for them. While these test exercises were being conducted, the participants were evaluated for proper technique. The participants then carried out the prescribed protocol for the first week of strength training; 3 sets of 10 repetitions of all the strengthening exercises i.e. scapular retraction, external rotation and shoulder flexion for lower trapezius. After the end of the third week the participants progressed to the next higher level of resistance using the theraband. This exercise progression was based on the work of Wang et al (1990) in which a similar protocol produced significant strength gains. The exercises were conducted three times per week.

The nature of stretching exercises were demonstrated and described to the participants. The first stretch for anterior chest muscles required the participant to assume a supine position on a 5-inch diameter from roll, which ran down the centre of the back. The subject's partner was asked to grasp the subject's shoulder and slowly press down in the direction of the floor until instructed to stop and hold for 30 seconds. This was repeated twice per training session. The second stretch for shoulder internal rotators required the subject to assume a kneeling position in front of his standing partner and lace his fingers behind the subject's scapulae, lacing his fingers together as well. The partner was asked to pull in a diagonal direction, both up and back from the subject's trunk, until instructed to stop. The stretch was held for 30 seconds and repeated twice per training session The change in forward head posture, forward shoulder posture, head to shoulder translation, head to ankle translation, shoulder to ankle translation and New York Postural Rating Scale (NYPRS) measurement was calculated (posttest score - pretest score) for both shoulders in each group. To determine the effectiveness of the intervention, the difference score was used to compare the two groups and statistical evaluation was done by using a student unpaired-t-test.

\section{Results and Discussion}

\begin{tabular}{cccccc}
\multicolumn{7}{c}{ Table 1: Demographic Variables } \\
\hline \multirow{2}{*}{ Variable } & $\begin{array}{c}\text { Control } \\
\text { Group }\end{array}$ & $\begin{array}{c}\text { Study } \\
\text { Group }\end{array}$ & $\begin{array}{c}\text { 't' } \\
\text { value }\end{array}$ & Df & $\begin{array}{c}\text { 'p' } \\
\text { value }\end{array}$ \\
\hline \multirow{2}{*}{ Age } & 19.07 & 18.8 & 0.35 & 28 & 0.728 \\
& \pm 2.02 & \pm 2.14 & & & \\
Height & 167.87 & 168.27 & 0.27 & 28 & 0.787 \\
& \pm 3.94 & \pm 4.10 & & & \\
Weight & 63.93 & 61.93 & 0.98 & 28 & 0.334 \\
& \pm 3.43 & \pm 7.09 & & & \\
BMI & 22.33 & 21.67 & 1.10 & 28 & 0.279 \\
& \pm 1.11 & \pm 2.06 & & &
\end{tabular}

The difference between the pretest and post test measurement of FHP, FSP, HST, HAT, SAT and NYPRS (shoulder component) are presented table 2. The interventional group showed statistically significant decrease in FSP (mean difference $-5.2 \pm 0.67$ as compared to $-1.4 \pm 0.50$, observed in control group $(\mathrm{p}=0.000)$, increase in NYPRS score (mean difference $3.46 \pm 0.91$ as compared to $0.67 \pm 0.97$, in control group $\mathrm{p}=$ 0.000 ). Student unpaired ' $t$ ' test was used as a test of significance to evaluate the intragroup effectiveness of the two treatment protocols under consideration in the present study. 
FORWARD SHOULDER POSTURE

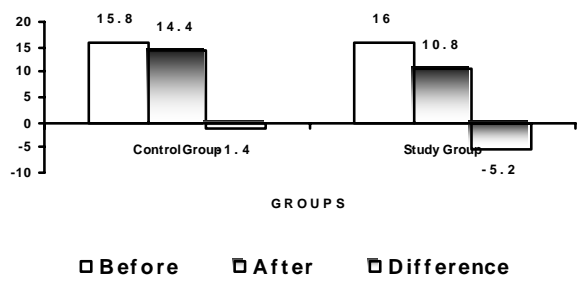

Figure 1: Effect of training on Forward Shoulder Posture

Table No. 2: Effect of training on study variables

\begin{tabular}{|c|c|c|c|c|c|c|}
\hline \multicolumn{2}{|c|}{ Parameters } & \multirow{2}{*}{$\begin{array}{c}\begin{array}{c}\text { Control } \\
\text { Group }\end{array} \\
\text { (Mean } \pm \text { SD) }\end{array}$} & \multirow{2}{*}{$\begin{array}{c}\begin{array}{c}\text { Study } \\
\text { Group } \\
\text { (Mean } \pm \text { SD) }\end{array} \\
\mathbf{8 . 4 0 \pm 0 . 7 4}\end{array}$} & \multirow{2}{*}{$\begin{array}{c}\begin{array}{c}\text { 't' } \\
\text { value }\end{array} \\
0.00\end{array}$} & \multirow{2}{*}{$\begin{array}{r}\text { df } \\
28\end{array}$} & \multirow{2}{*}{$\begin{array}{c}\begin{array}{c}\text { ‘p' } \\
\text { value }\end{array} \\
1.00\end{array}$} \\
\hline & Pre & & & & & \\
\hline \multirow{3}{*}{$\stackrel{\mathbf{I}}{\mathbf{I}}$} & Post & $8.40 \pm 0.74$ & $8.40 \pm 0.74$ & 0.00 & 28 & 1.00 \\
\hline & Diff & 0.00 & 0.00 & - & 28 & - \\
\hline & Pre & $15.80 \pm 0.68$ & $16.00 \pm 0.93$ & 00.67 & 28 & 0.51 \\
\hline \multirow[t]{3}{*}{ के } & Post & $14.40 \pm 0.74$ & $10.80 \pm 0.77$ & 13.04 & 28 & $0.00 *$ \\
\hline & Diff & $-1.40 \pm 0.50$ & $-5.2 \pm 0.67$ & 17.41 & 28 & $0.00 *$ \\
\hline & Pre & $7.40 \pm 0.74$ & $7.60 \pm 1.06$ & 00.60 & 28 & 0.55 \\
\hline \multirow[t]{3}{*}{ 氞 } & Post & $6.00 \pm 0.76$ & $2.40 \pm 1.06$ & 10.73 & 28 & $0.00 *$ \\
\hline & Diff & $-1.40 \pm 0.50$ & $-5.20 \pm 0.67$ & 17.41 & 28 & $0.000^{*}$ \\
\hline & Pre & $4.13 \pm 0.83$ & $4.33 \pm 1.05$ & 00.57 & 28 & 0.57 \\
\hline \multirow[t]{3}{*}{$\stackrel{5}{5}$} & Post & $4.13 \pm 0.83$ & $4.33 \pm 1.05$ & 00.57 & 28 & 0.57 \\
\hline & Diff & 0.00 & 0.00 & - & 28 & - \\
\hline & Pre & $11.53 \pm 0.92$ & $11.93 \pm 1.10$ & 1.08 & 28 & 0.29 \\
\hline \multirow[t]{3}{*}{ है } & Post & $10.13 \pm 0.99$ & $6.73 \pm 0.80$ & 10.34 & 28 & $0.00 *$ \\
\hline & Diff & $-1.40 \pm 0.50$ & $-5.20 \pm 0.67$ & 17.41 & 28 & $0.00 *$ \\
\hline & Pre & $1.00 \pm 0.00$ & $1.40 \pm 0.83$ & 1.87 & 28 & 0.07 \\
\hline \multirow{2}{*}{$\begin{array}{l}\tilde{v} \\
\stackrel{\tilde{z}}{2} \\
z\end{array}$} & Post & $1.67 \pm 0.98$ & $4.87 \pm 0.52$ & 11.22 & 28 & $0.00 *$ \\
\hline & Diff & $0.67 \pm 0.97$ & $3.46 \pm 0.91$ & 8.10 & 28 & $0.00 *$ \\
\hline
\end{tabular}

HEAD TO SHOULDER TRANSLATION
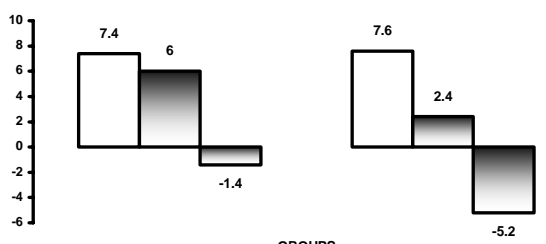

GROUPS

QBefore $\mathbf{Q}$ After QDifference

Figure 2: Effect of training on Head to Shoulder Translation SHOULDER TO ANKLE TRANSLATION

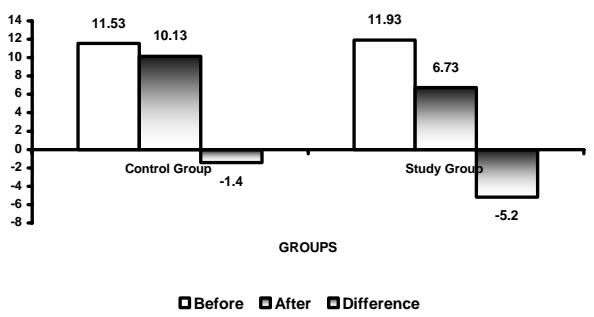

Figure 3: Effect of training on Shoulder to Ankle Translation

Discussion

The present study showed that six weeks stretching and strengthening exercise program of shoulder muscles along with normal training would be of benefit in correcting the forward shoulder posture in Cricket fast bowlers. There was significant difference in forward shoulder posture, head to shoulder translation, shoulder to ankle translation and New York postural rating scale (shoulder component) on the forty-second day as compared to the first day. Based on the theories of muscle imbalance, clinicians postulate that strengthening of the posterior scapular stabilizers combined with stretching of pectoral muscles can correct posture and muscle imbalance and can alter scapulohumeral rhythm thus helpful in promoting shoulder injuries Wang et al (1990).

The exercise progression used in this study was based on the work of Wang 
et al in which a similar exercise protocol of stretching of tight muscles and strengthening of weak muscles produce significant strength gains, reducing the shoulder injuries thus by correcting the posture of the shoulder. Rozier and Schafer (1981) compared the maximum isometric and isotonic muscle torques before and after six-week exercise program (three times per week and daily for five times per week). They showed that the strength gains were significant in both groups.

Shoulder impingement syndrome may result form altered shoulder posture and decreased scapular upward rotation. This belief is based on the notion that when the shoulder is abducted overhead with insufficient scapular upward rotation, the greater tuberosity and acromian becomes too close and therefore the soft tissues in the subacromial space may be pinched by the bony structures. Based on this idea exercises focusing on scapular muscles must be prescribed to improve the scapular upward rotation thus improving the posture of the shoulder (Piane and Voight, 1993). Weak muscles cannot be strengthened if their antagonist counterparts are not stretched. Thus it is important to stretch anterior chest muscles, such as pectoralis major and minor before starting the strengthening exercise protocol for scapular muscles to correct the rounded shoulder posture.

Studies with different population, using a different series of strengthening exercises showed an increase in strength and correcting shoulder posture (Lukasiewicz et al, 1999). Combining stretching of the hypertrophied anterior shoulder muscles with strengthening of relatively weaker posterior muscles might have a significant synergistic effect on shoulder posture. A stretching program that requires a 30 second hold and repeats each exercise two times has been demonstrated to increase the muscle length in hamstrings (Bandy et al, 1997). This focused stretching routine, aimed at lengthening the shoulders internal rotator and adductor muscles combined with the strengthening exercise improves the posture of the shoulder.

\section{Conclusion}

Shoulder stretching and strengthening exercises are effective in reducing the muscular imbalance and correcting the forward shoulder posture in cricket fast bowlers. Thus shoulder stretching and strengthening exercises should be incorporated in the regular training of cricket fast bowlers to prevent shoulder injuries.

\section{References}

Arnold, C.M., Beatty, B., Harrison, L.E., Obszynski, W. 2000. The reliability of fine clinical postural alignment measures for women with osteoporosis. Physiotherapy Canada, Fall: 28694

Bandy, W.D., Irion, J.M., Briggler, M. 1997. The effort of time and frequency of static stretching on flexibility of hamstrings muscles. Phys. Ther., 77: $1090-6$

Brasch, R. 1971. How did sports begin? Camberwell: Longman;

Honcock, R.E., Hawkins, R.J. 1996. Application of electromyography in the throwing shoulder. Clin. Orthopedics, 330: 84-97.

Kendall, F.P., McCreary, E.K., Provance, P.G. 1993. Muscles: Testing and function. $4^{\text {th }}$ ed.: Baltimore Md: Williams and Wilkins.

Kluemper, M., Uhl, T., Hazetrigg, M. 2006. Effects of stretching and strengthening shoulder muscles on forward shoulder posture in competitive swimmers. J. Sports Rehabil., 15: 56-70.

Lukasiewicz, A.C., McClure, P., Michoner, L., Pratt, N., Sannett, B.. 1999. Comparison of 3-dimensional scapular position and orientation between subjects with and without shoulder impingement. J. Orthop. Sports Phys. Ther., 29: 594-86.

Piane, R.M., Voight, M. 1993. The role of the scapula. J. Orthop. Sports Phys. Ther., 18: 386-91. 
Rozier, C.K., Schafer, D.S. 1981. Isokinematic strength training: Comparison of daily and three times weekly patterns. Int. J. Rehabil. Res., 4: 345-51.

Sahrmann, S.A. 2002. Diagnosis and Treatment of movement impairment syndromes. St. Louis, Mo: Mosby;

Stretch, R.A. 1995. The incidence and nature of injuries in schoolboy cricketers. S. Afr. Med. J., 85: 11824.

Stretch, R.A. 2001. Incidence and nature of epidemiological injuries to elite South African cricket players. S. Afr. Med., 91: 336-9.
Voight, M.L., Thomson, B.C. 2000. The role of scapula in the rehabilitation of shoulder injuries. J. Athl. Train., 35(3): 364-73.

Wang, C.H., McCreay, P., Praft, N., Nobilini, R. 1990. Stretching and strengthening exercises: their effect on three-dimensional scapular kinematics. Arch. Phys. Med. Rehabil., 80: 923-9.

Weightman, D., Browne, R.C. 1971. Injuries in eleven selected sports. Br. J. Sports Med., 2: 27. 